\title{
Streptococcus mutans diacylglycerol kinase homologue: a potential target for anti-caries chemotherapy
}

\section{Correspondence \\ Yoshihisa Yamashita \\ yoshi@dent.kyushu-u.ac.jp}

Received 14 September 2010 Accepted 6 February 2011

\section{Yukie Shibata, ${ }^{1}$ Miki Kawada-Matsuo, ${ }^{1} \dagger$ Yasuhito Shirai, ${ }^{2}$ Naoaki Saito, ${ }^{2}$ Dan $\mathrm{Li}^{1}$ and Yoshihisa Yamashita ${ }^{1}$}

\author{
${ }^{1}$ Section of Preventive and Public Health Dentistry, Division of Oral Health, Growth and \\ Development, Kyushu University Faculty of Dental Science, Fukuoka, Japan \\ ${ }^{2}$ Biosignal Research Center, Kobe University, Kobe, Japan
}

\section{INTRODUCTION}

Streptococcus mutans, the major aetiological factor in human dental caries, is capable of forming the biofilm commonly known as 'dental plaque' on the surfaces of teeth (Loesche, 1986; Tanzer et al., 2001). Within the dental plaque, $S$. mutans synthesizes large amounts of acids from fermentable dietary carbohydrates. Acid accumulation can eventually cause the teeth's hard, crystalline structure to be dissolved, resulting in the formation of carious lesions (Quivey et al., 2001). S. mutans has the ability to tolerate the typically low $\mathrm{pH}$ of dental plaque and continue to produce acids, contributing to its cariogenicity. The tolerance of low $\mathrm{pH}$ is considered to be one of this micro-organism's most important virulence factors.

Diacylglycerol kinase (Dgk) is an enzyme that phosphorylates diacylglycerol (DG) to produce phosphatidic acid. In eukaryotic cells, both DG and phosphatidic acid are important lipid second messengers that regulate the

tPresent address: Department of Oral Microbiology, Kagoshima University Graduate School of Medical and Dental Sciences, Kagoshima, Japan.

Abbreviations: DG, diacylglycerol; Dgk, diacylglycerol kinase; PSL, photostimulated luminescence.

A table of primers is available as supplementary material with the online version of this paper. expression of a number of enzymes. Therefore, Dgk plays an important role in regulating many intracellular signalling pathways (Kanoh et al., 2002; Mérida et al., 2008; Topham \& Prescott, 1999; Van Blitterswijk \& Houssa, 2000). In bacteria, Dgk functions in the recycling of DG produced during the turnover of membrane phospholipids (Hasin \& Kennedy, 1982; Rotering \& Raetz, 1983), and similar to eukaryotic Dgk, it also plays an important role in microbial physiology under conditions of environmental stress (Raetz \& Newman, 1979; Walsh et al., 1986; Yamashita et al., 1993).

Many researchers have sought to explain the mechanisms of acid tolerance in S. mutans, and various genes contributing to this property have been identified. In a previous study (Shibata et al., 2009), we reported that the C-terminal tail of a Dgk homologue was indispensable for tolerance to acid stress in S. mutans and that the Dgk homologue's kinase activity was closely related to the acid tolerance of this organism. Furthermore, a dgk deletion mutant isolate exhibited markedly reduced levels of smooth-surface carious lesions in pathogen-free rats (Shibata et al., 2009). Inhibitors of the Dgk homologue's enzymic activity may therefore be useful in attenuating the virulence of $S$. mutans.

In this study, we generated mutations in genes that have been previously reported to be involved in acid tolerance in 
S. mutans and examined their contribution to acid tolerance, in order to determine the most desirable target for anti-caries agents. Additionally, we investigated the effects of inhibitors of the putative candidate on the acid tolerance of $S$. mutans, to assess their potential usefulness as anti-caries agents.

\section{METHODS}

Bacterial strains and culture conditions. Escherichia coli strain RZDGK11 producing full-size S. mutans Dgk protein was constructed through transformation of the mutant strain RZ6, which lacks a functional E. coli dgk gene, as described previously (Shibata et al., 2009). E. coli strains and S. mutans strains were maintained and grown routinely as described previously (Shibata et al., 2002). Antibiotics were used at the following concentrations: $200 \mu \mathrm{g}$ erythromycin $\mathrm{ml}^{-1}$ or $50 \mu \mathrm{g}$ ampicillin $\mathrm{ml}^{-1}$ for E. coli; $10 \mu \mathrm{g}$ erythromycin $\mathrm{ml}^{-1}$ for $S$. mutans.

DNA manipulation. Standard DNA recombinant procedures, such as DNA isolation, endonuclease restriction, ligation and agarose gel electrophoresis were carried out as described by Sambrook \& Russell (2001). Transformation of S. mutans and E. coli was performed as described elsewhere (Hanahan, 1983; Perry et al., 1983).

Construction of the mutant forms of the genes that contribute to aciduricity in S. mutans. In this study, we created $S$. mutans UA159 mutant strains carrying mutated forms of the following 15 genes: aguB (Griswold et al., 2004), brpA (Wen et al., 2006), ciaH (Ahn et al., 2006), clpP (Lemos \& Burne, 2002), dltC (Boyd et al., 2000), ffh (Kremer et al., 2001), ftsY (Hasona et al., 2005), glrA (Cvitkovitch et al., 2000), gluA (Yamashita et al., 1998), htrA (Biswas \& Biswas, 2005), lgl (Korithoski et al., 2007), luxS (Wen \& Burne, 2004), ropA (Wen et al., 2005), uvrA (Hanna et al., 2001) and yidC2 (Dong et al., 2008). The $d l t C$ and ciaH genes were replaced by an erythromycin resistance gene using double cross-over homologous recombination, as described previously (Kawada-Matsuo et al., 2009). The other mutants were generated through the interruption of linear target gene fragments by the same erythromycin resistance gene, inserted at appropriate restriction sites, using double cross-over homologous recombination. Correct insertions or replacements in transformants were confirmed by PCR. The mutant UADGK1 (Shibata et al., 2009), in which the C-terminal 10 amino acid residues of Dgk were deleted, was used as a $d g k$ mutant. The primers used to generate and confirm the identities of the mutants are listed in Supplementary Table S1 (available with the online journal).

Evaluation of the acid sensitivity of the strains. The 16 mutant strains and the wild-type control UA159 strain cells were grown in brain heart infusion broth (BHI; Difco) overnight at $37{ }^{\circ} \mathrm{C}$ in $5 \%$ $\mathrm{CO}_{2}$. The cultures were then diluted $1: 10$ into fresh $\mathrm{BHI}$ and grown to an $\mathrm{OD}_{550}$ of $\sim 0.5$. Aliquots $(20 \mu \mathrm{l})$ of the cell suspensions having the same turbidity were inoculated into wells that contained $200 \mu \mathrm{l}$ fresh BHI medium adjusted to either $\mathrm{pH} 7.4$ or 5.5 with $50 \mathrm{mM}$ sodium acetate buffer. Growth was monitored by measuring the optical density at $550 \mathrm{~nm}$ using a Spectramax 340 $\mathrm{PC}^{384}$ microplate spectrophotometer (Molecular Devices). Wells containing only BHI were used as controls. Growth curves were generated from the data obtained from three independent experiments. In addition, each strain was grown at $\mathrm{pH} 7.4$ or 5.5 , as described above, and incubated for one additional hour after reaching the maximum $\mathrm{OD}_{550}$. The cultures were then serially diluted, plated on BHI agar plates, and incubated at $37{ }^{\circ} \mathrm{C}$ in $5 \% \mathrm{CO}_{2}$ for 2 days before colonies were counted. The mutant/wild-type c.f.u. ratio was calculated. Three independent experiments were performed in triplicate.
Growth inhibition assay. The Dgk inhibitors, 6-\{2-\{4[( $p$-fluorophenyl)phenylmethylene]-1-piperidinyl $\}$ ethyl $\}$-7-methyl5H-thiazolo(3,2-a)pyrimidine-5-one (R59022) and 3-\{2-\{4[bis-(4fluorophenyl)methylene]-1-piperidinyl $\}$ ethyl $\}$-2,3-dihydro-2-thioxo$4(1 \mathrm{H})$ quinazolinone (R59949) were purchased from Merck. To examine the effects of R59022 and R59949 on the growth of S. mutans, wild-type UA159 cells were inoculated into microtitre plate wells, each containing $200 \mu \mathrm{l}$ fresh BHI medium ( $\mathrm{pH} 7.4,5.5,5.4,5.3,5.2$, 5.1 or 5.0$)$ containing R59022 $(100 \mu \mathrm{M})$, R59949 $(25 \mu \mathrm{M})$ or DMSO (control). After incubation at $37{ }^{\circ} \mathrm{C}$ in $5 \% \mathrm{CO}_{2}$ for $16 \mathrm{~h}$ the $\mathrm{OD}_{550}$ was measured. Three independent experiments were performed in triplicate.

Measurement of intracellular pH. Intracellular $\mathrm{pH}$ measurements were performed as described by Sawatari \& Yokota (2007). Briefly, cells were cultured until mid-exponential phase, harvested and washed twice with buffer A $[150 \mathrm{mM}$ potassium phosphate $(\mathrm{pH} 6.5), 1 \mathrm{mM} \mathrm{MgSO} 4$ ]. The cells were resuspended to an $\mathrm{OD}_{550}$ value of 0.5 in buffer $\mathrm{A}$ and incubated at $37^{\circ} \mathrm{C}$ for $30 \mathrm{~min}$ in the presence of carboxyfluorescein diacetate succinimidyl ester (cFDA SE; Molecular Probes), a fluorescent $\mathrm{pH}$ probe. To eliminate unbound probe, the cells were incubated with glucose for $1 \mathrm{~h}$ and then washed once in buffer $\mathrm{A}$. The cells were subsequently resuspended in buffer $\mathrm{B}$ (buffer A supplemented with $100 \mathrm{mM}$ 2-morpholinoethanesulfonic acid), and the intracellular $\mathrm{pH}$ was measured. Three independent experiments were performed.

Kinase assay. A kinase assay was conducted as described previously (Shibata et al., 2009). Kinase activity in cell lysates was examined by an octylglucoside mixed-micelle assay (Preiss et al., 1986), using undecaprenol (Larodan Fine Chemicals) as a substrate. The synthesized undecaprenylphosphate, radiolabelled by $\left[\gamma^{32} \mathrm{P}\right] \mathrm{ATP}$, was separated on silica gel 60 TLC plates (Merck), detected by BAS2500 (Fujix), and finally represented as photostimulated luminescence (PSL). For the kinase inhibition assay, the Dgk inhibitors were used at a concentration of $100 \mu \mathrm{M}$. Three independent experiments were performed.

\section{RESULTS AND DISCUSSION}

\section{Mutant strain acid sensitivity}

To examine the effects of the mutations on S. mutans acid tolerance, the 16 mutant strains and the wild-type control UA159 strain cells were grown in BHI broth adjusted to pH 7.4 or 5.5 (Fig. 1, Table 1). At pH 7.4, the brpA, ciaH, $d g k, d l t C, h t r A, \operatorname{lgl}, \operatorname{luxS}$, ropA and uvrA mutant cells grew similarly to the wild-type UA159 cells, whereas the other mutant strains, particularly the gluA and yidC2 mutant strains, grew more slowly than the wild-type strain (Fig. 1a). Inactivation of $a g u B, \operatorname{brp} A, g \operatorname{lr} A, h \operatorname{tr} A, \operatorname{lgl}, \operatorname{lux} S, \operatorname{rop} A$, or $u v r A$ did not significantly affect the acid tolerance of $S$. mutans, as assessed by growth at pH 5.5 (Fig. 1b). In contrast, the $c i a H, c l p P, d g k, d l t C$, ffh, fts $Y$, gluA and yidC2 mutant strains displayed significantly reduced growth rates. Notably, the $d g k$ and the gluA mutant strains grew extremely slowly at $\mathrm{pH} 5.5$. Not all mutant strains grew at rates similar to that of wild-type UA159 cells, even at $\mathrm{pH}$ 7.4. Calculation of the mutant/wild-type strain c.f.u. ratio at $\mathrm{pH} 5.5$ revealed that the inactivation of the $d g k$ or $g l u A$ gene markedly attenuated the aciduricity of $S$. mutans (Table 1). The growth data of all strains tested are 
(a)

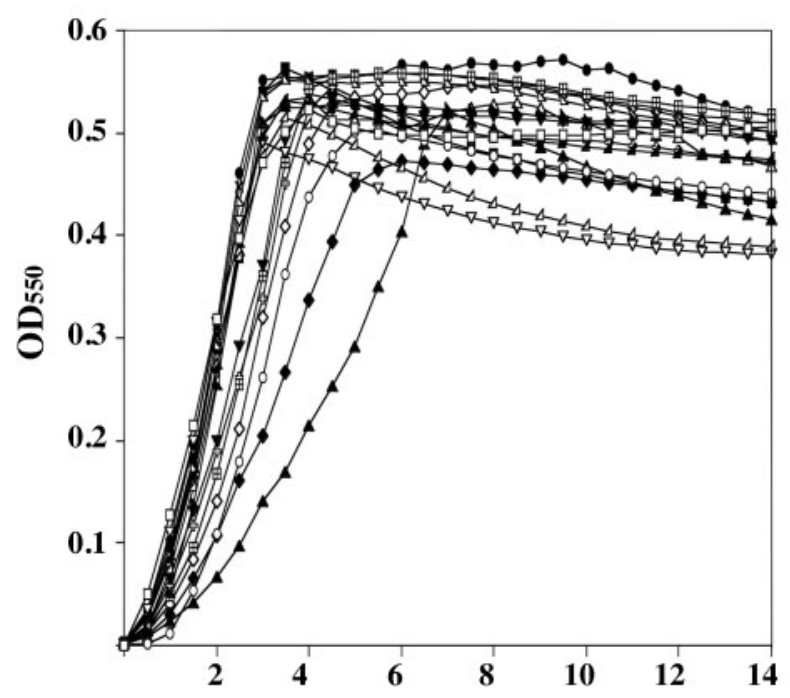

(b)

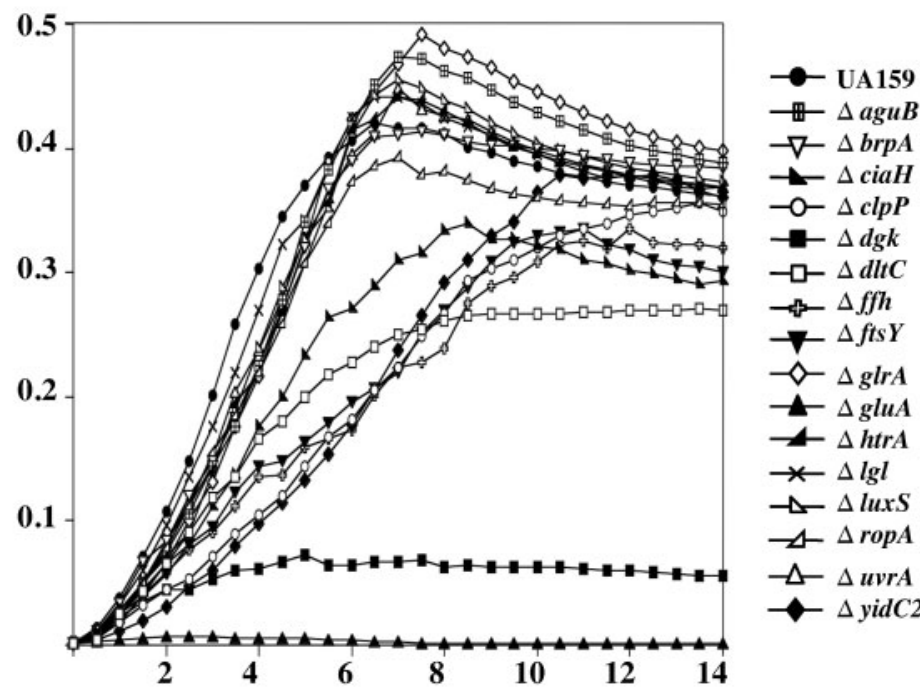

Time (h)

Fig. 1. Growth curves of S. mutans UA159 (wild-type) and mutant strains grown in BHI medium at pH 7.4 (a) or 5.5 (b). Growth was defined in terms of increased $\mathrm{OD}_{550}$ and was calculated by subtracting the initial $\mathrm{OD}_{550}$ value from those values recorded after the indicated periods of growth. Data represent the means of three independent experiments.

Table 1. Growth data of S. mutans UA159 and 16 mutant strains at $\mathrm{pH} 7.4$ and 5.5

Doubling time was calculated based on the formula $\ln \mathrm{Z}-\ln \mathrm{Z}_{0}=k\left(t-t_{0}\right)$, where $k$ is the growth rate, and $g=0.693 / k$, where $g$ is the doubling time. The c.f.u. ratio was calculated between the mutant (MT) and wild-type UA159 (WT) strains. Values are the mean [ \pm sD] of three independent experiments.

\begin{tabular}{|c|c|c|c|c|c|c|c|c|}
\hline \multirow[t]{2}{*}{ Strain } & \multicolumn{4}{|c|}{ pH 7.4} & \multicolumn{4}{|c|}{ pH 5.5} \\
\hline & $\begin{array}{l}\text { Doubling } \\
\text { time (min) }\end{array}$ & $\begin{array}{c}\text { Maximum } \\
\mathrm{OD}_{550}\end{array}$ & $\begin{array}{l}\text { Final } \\
\text { OD }_{550}\end{array}$ & $\begin{array}{l}\text { c.f.u. ratio } \\
(\%, \text { MT/WT })\end{array}$ & $\begin{array}{l}\text { Doubling } \\
\text { time (min) }\end{array}$ & $\begin{array}{c}\text { Maximum } \\
\mathrm{OD}_{550}\end{array}$ & $\begin{array}{l}\text { Final } \\
\text { OD }_{550}\end{array}$ & $\begin{array}{c}\text { c.f.u. ratio } \\
(\%, \text { MT/WT })\end{array}$ \\
\hline UA159 & 55.9 [3.3] & $0.59[0.08]$ & $0.52[0.08]$ & 100 & $109.3[7.2]$ & $0.43[0.06]$ & $0.36[0.03]$ & 100 \\
\hline$\Delta a g u B$ & 55.7 [4.9] & $0.57[0.03]$ & $0.52[0.05]$ & $84.2[10.1]$ & $110.8[22.9]$ & $0.49[0.03]$ & $0.39[0.01]$ & 196.3 [31.7] \\
\hline$\Delta \operatorname{brp} A$ & $50.0[4.5]$ & $0.49[0.07]$ & $0.38[0.06]$ & $72.9[8.3]$ & $127.1[12.4]$ & $0.43[0.03]$ & $0.38[0.02]$ & $172.8[7.6]$ \\
\hline$\Delta d g k$ & $46.8[4.0]$ & $0.57[0.07]$ & $0.43[0.04]$ & $89.9[13.4]$ & $>1000$ & $0.08[0.02]$ & $0.06[0.02]$ & $\begin{array}{r}87.2 \times 10^{-2} \\
{\left[10.9 \times 10^{-2}\right]}\end{array}$ \\
\hline$\Delta d l t C$ & $56.6[2.4]$ & $0.52[0.05]$ & $0.51[0.04]$ & $92.1[12.2]$ & $171.4[4.9]$ & $0.27[0.04]$ & $0.27[0.04]$ & 41.2 [12.4] \\
\hline$\Delta f f h$ & $62.5[1.1]$ & $0.55[0.04]$ & $0.47[0.02]$ & $78.1[9.1]$ & $178.5[4.0]$ & $0.34[0.03]$ & $0.32[0.02]$ & $53.5[6.8]$ \\
\hline$\Delta f t s Y$ & $68.3[5.5]$ & $0.54[0.04]$ & 0.49 [0.04] & 73.2 [8.9] & $169.0[11.5]$ & $0.34[0.02]$ & $0.30[0.02]$ & $63.5[9.2]$ \\
\hline$\Delta \operatorname{lgl}$ & 46.3 [3.9] & $0.56[0.04]$ & $0.51[0.03]$ & 83.5 [7.6] & 121.0 [11.9] & $0.45[0.04]$ & $0.37[0.00]$ & $170.4[26.8]$ \\
\hline$\Delta \operatorname{luxS}$ & $46.6[3.7]$ & $0.56[0.04]$ & $0.50[0.07]$ & 81.2 [11.4] & $121.8[18.1]$ & $0.47[0.02]$ & $0.37[0.00]$ & $185.8[25.1]$ \\
\hline$\triangle \operatorname{rop} A$ & $51.5[0.5]$ & $0.52[0.04]$ & $0.39[0.04]$ & $61.4[6.0]$ & $120.3[0.8]$ & $0.40[0.04]$ & $0.36[0.04]$ & $85.3[7.0]$ \\
\hline$\Delta u v r A$ & $49.5[8.6]$ & $0.55[0.06]$ & $0.47[0.08]$ & $83.7[5.1]$ & $116.8[4.4]$ & $0.45[0.00]$ & $0.37[0.02]$ & $82.1[8.0]$ \\
\hline$\Delta$ yidC2 & 81.1 [9.6] & $0.47[0.07]$ & $0.43[0.06]$ & $49.9[8.5]$ & $176.3[5.6]$ & $0.40[0.03]$ & $0.36[0.03]$ & $118.0[24.5]$ \\
\hline
\end{tabular}


summarized in Table 1 . The gluA mutant strain exhibited a reduced growth rate at both neutral and acidic $\mathrm{pH}$, and thus we focused on $d g k$ as a gene responding specifically to $\mathrm{pH}$ reduction in S. mutans.

\section{Effects of Dgk inhibitors on the growth of $S$. mutans}

Mammalian Dgks have been extensively studied, and several compounds that inhibit their activity have been identified. R59022 and R59949 are the most commonly used inhibitors, and both interact directly with Dgk. R59022 inhibits Dgk without affecting phosphodiesterase or phosphatidylinositol kinase (De Chaffoy de Courcelles et al., 1985). R59949 acts on the enzyme's catalytic domain (Jiang et al., 2000). In contrast, the inhibition of prokaryotic Dgk enzymes has not been studied. We therefore evaluated the effects of R59022 and R59949 on the growth of $S$. mutans. Both inhibitors can normally be used at a concentration of $100 \mu \mathrm{M}$ in the hydrophobic conditions of enzyme assays. However, in the growth assay, R59949 did not fully dissolve in BHI medium (hydrophilic) at a concentration of $100 \mu \mathrm{M}$, although R59022 did. Thus, R59022 and R59949 were used at concentrations of 100 and $25 \mu \mathrm{M}$, respectively, in the growth inhibition assay. We confirmed that the addition of the inhibitors did not affect the $\mathrm{pH}$ of the buffered BHI medium. While neither R59022 nor R59949 influenced the growth of $S$. mutans at pH 7.4, R59949 significantly inhibited the growth of $S$. mutans at acidic pH (Fig. 2a). R59022 had no effect on S. mutans growth rate (Fig. 2b). The inhibition ratio of R59949 increased with decreasing $\mathrm{pH}$, with ratios of $13,29,58,68$ and $78 \%$ at $\mathrm{pH} 5.4,5.3,5.2,5.1$ and 5.0 , respectively.

\section{Effect of the Dgk inhibitors on the enzymic activity of S. mutans Dgk}

The effects of R59022 and R59949 on the kinase activity of $S$. mutans Dgk were assayed. Cell lysates obtained from E. coli RZDGK11 expressing full-length $S$. mutans Dgk protein were used as crude $S$. mutans Dgk samples in a kinase activity assay. It has been shown that cell lysates from E. coli RZ6, the parent strain of RZDGK11, do not exhibit any kinase activity when provided with undecaprenol as a substrate (Shibata et al., 2009). We first confirmed that the addition of the inhibitors did not affect the $\mathrm{pH}$ of the reaction mixture for kinase activity assay. Neither R59022 nor R59949 affected Dgk kinase activity at pH 7.4 (data not shown), consistent with the above observations that the inhibitors did not affect the growth rate of $S$. mutans at neutral $\mathrm{pH}$.

As intracellular $\mathrm{pH}$ is not necessarily equal to that of the local environment, we determined the intracellular $\mathrm{pH}$ of $S$. mutans at various external $\mathrm{pH}$ values. The intracellular $\mathrm{pH}$ values of $S$. mutans were $6.57 \pm 0.07,6.51 \pm 0.07,6.45 \pm$ $0.07,6.38 \pm 0.07,6.32 \pm 0.07$ and $6.26 \pm 0.07$ at external $\mathrm{pH}$ values of $5.5,5.4,5.3,5.2,5.1$ and 5.0 , respectively. Moreover, the addition of R59949 did not affect the intracellular $\mathrm{pH}$. Considering that $\mathrm{R} 59949$ showed greater than $50 \%$ inhibition of $S$. mutans growth at $\mathrm{pH} 5.2$, the kinase inhibition experiment was performed at $\mathrm{pH}$ 6.4. The kinase activities in E. coli RZDGK11 cell lysates using undecaprenol as a substrate were $37.3 \pm 2.9 \mathrm{PSL} \mathrm{mm}^{-2}$ and $29.4 \pm 4.3$ PSL $\mathrm{mm}^{-2}$, respectively, in the absence and presence of R59949, reflecting an inhibition of about $20 \%$ $(P<0.05$, Student's $t$-test) by R59949. In contrast, R59022 did not inhibit kinase activity under these conditions (data not shown). (a)

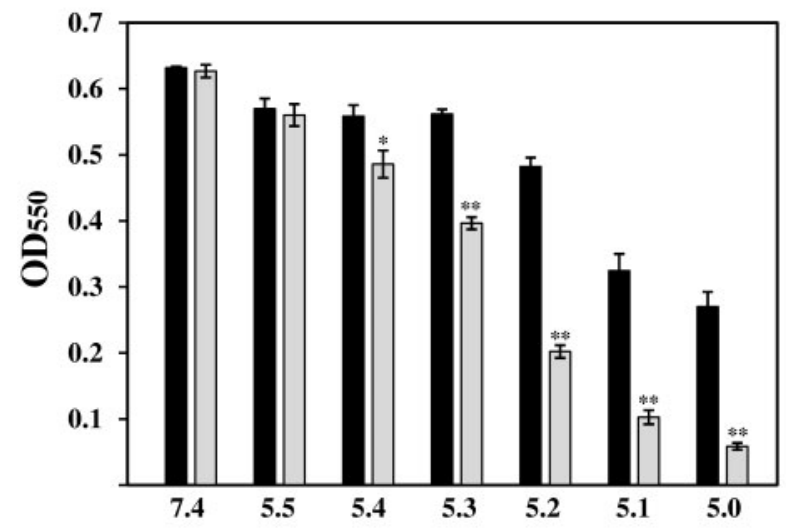

(b)

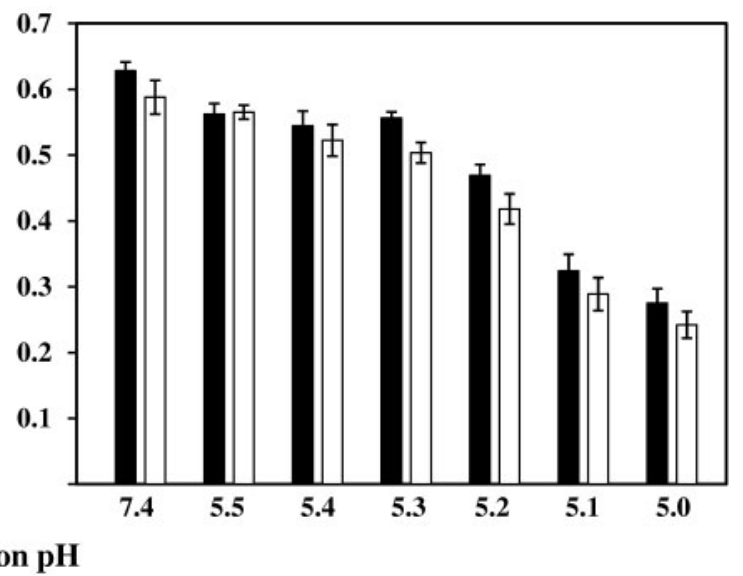

Fig. 2. Effect of R59949 (a) and R59022 (b) on the growth of $S$. mutans. The $y$-axis represents the $\mathrm{OD}_{550}$ after $16 \mathrm{~h}$ of incubation. Data are the means \pm SDS of three independent experiments. Differences in growth rate between cells cultured in the presence and absence of Dgk inhibitor were analysed using Student's $t$-test: ${ }^{*}, P<0.05 ;{ }^{\star *}, P<0.0001$. Black bars, DMSO; grey bars, R59949; white bars, R59022. 
Collectively, these results support the previous conclusion that Dgk kinase activity is closely related to $S$. mutans acid tolerance. However, the inhibitory effect of R59949 on Dgk kinase activity was not as strong as expected. In our previous study (Shibata et al., 2009), S. mutans UADGK6 expressing Dgk6, a truncated form of Dgk lacking 5 Cterminal amino acids, exhibited 40 and $60 \%$ reduced growth at pH 5.5 and 5.4, respectively, compared with $S$. mutans expressing full-length Dgk. However, R59949 had no effect on the growth of $S$. mutans at $\mathrm{pH} 5.5$ and caused significant growth inhibition $(>50 \%)$ at $\mathrm{pH}$ 5.2. This indicates that the effect of R59949 was weaker than that of the C-terminal truncation of Dgk. Thus, given that Dgk6 displayed $60 \%$ reduced kinase activity with undecaprenol as a substrate, compared with full-length Dgk, the calculated $20 \%$ reduction in Dgk kinase activity caused by $\mathrm{R} 59949$ seems reasonable.

Unlike eukaryotic Dgks, bacterial Dgks, including that of $S$. mutans, are small proteins exhibiting minimal amino acid sequence similarity with their eukaryotic homologues. Moreover, the principal substrate for S. mutans Dgk is undecaprenol, not DG (Lis \& Kuramitsu, 2003). Nevertheless, R59949, an inhibitor of mammalian Dgks, inhibited the Dgk activity and acid tolerance of $S$. mutans. A second inhibitor of mammalian Dgks, R59022, produced no such effect. Both R59022 and R59949 are known to selectively inhibit type I Dgks (De Chaffoy de Courcelles et al., 1985; Jiang et al., 2000), although their inhibitory effects on different Dgk isoforms have not been comprehensively evaluated. Thus, a few (minor) differences in isoform selectivity between R59949 and R59022 may exist. Moreover, differences in the molecular structures of R59949 and R59022 may contribute to the difference in their effectiveness against prokaryotic Dgks. It is not clear why an inhibitor of mammalian Dgks also inhibited Dgk activity in $S$. mutans. However, our study clearly shows that the addition of R59949 caused a reduction in aciduricity, one of the main virulence factors of $S$. mutans. Unfortunately, the direct use of R59949 in patients is not realistic. The development of more potent Dgk inhibitors specific for prokaryotic enzymes, through specific modifications based on comparisons of the molecular structures of R59949 and R59022, may lead to the discovery of new anti-caries agents.

\section{REFERENCES}

Ahn, S. J., Wen, Z. T. \& Burne, R. A. (2006). Multilevel control of competence development and stress tolerance in Streptococcus mutans UA159. Infect Immun 74, 1631-1642.

Biswas, S. \& Biswas, I. (2005). Role of HtrA in surface protein expression and biofilm formation by Streptococcus mutans. Infect Immun 73, 6923-6934.

Boyd, D. A., Cvitkovitch, D. G., Bleiweis, A. S., Kiriukhin, M. Y., Debabov, D. V., Neuhaus, F. C. \& Hamilton, I. R. (2000). Defects in D-alanyl-lipoteichoic acid synthesis in Streptococcus mutans results in acid sensitivity. J Bacteriol 182, 6055-6065.
Cvitkovitch, D. G., Gutierrez, J. A., Behari, J., Youngman, P. J., Wetz, J. E., Jr, Crowley, P. J., Hillman, J. D., Brady, L. J. \& Bleiweis, A. S. (2000). Tn917-lac mutagenesis of Streptococcus mutans to identify environmentally regulated genes. FEMS Microbiol Lett 182, 149-154.

De Chaffoy de Courcelles, D., Roevens, P. \& Van Belle, H. (1985). R 59 022, a diacylglycerol kinase inhibitor. Its effect on diacylglycerol and thrombin-induced $\mathrm{C}$ kinase activation in the intact platelet. J Biol Chem 260, 15762-15770.

Dong, Y., Palmer, S. R., Hasona, A., Nagamori, S., Kaback, H. R., Dalbey, R. E. \& Brady, L. J. (2008). Functional overlap but lack of complete cross-complementation of Streptococcus mutans and Escherichia coli YidC orthologs. J Bacteriol 190, 2458-2469.

Griswold, A. R., Chen, Y. Y. \& Burne, R. A. (2004). Analysis of an agmatine deiminase gene cluster in Streptococcus mutans UA159. J Bacteriol 186, 1902-1904.

Hanahan, D. (1983). Studies on transformation of Escherichia coli with plasmids. J Mol Biol 166, 557-580.

Hanna, M. N., Ferguson, R. J., Li, Y. H. \& Cvitkovitch, D. G. (2001). $u v r A$ is an acid-inducible gene involved in the adaptive response to low pH in Streptococcus mutans. J Bacteriol 183, 5964-5973.

Hasin, M. \& Kennedy, E. P. (1982). Role of phosphatidylethanolamine in the biosynthesis of pyrophosphoethanolamine residues in the lipopolysaccharide of Escherichia coli. J Biol Chem 257, 12475-12477.

Hasona, A., Crowley, P. J., Levesque, C. M., Mair, R. W., Cvitkovitch, D. G., Bleiweis, A. S. \& Brady, L. J. (2005). Streptococcal viability and diminished stress tolerance in mutants lacking the signal recognition particle pathway or YidC2. Proc Natl Acad Sci U S A 102, 1746617471.

Jiang, Y., Sakane, F., Kanoh, H. \& Walsh, J. P. (2000). Selectivity of the diacylglycerol kinase inhibitor 3-[2-(4-[bis-(4-fluorophenyl)methylene]-1-piperidinyl)ethyl]-2, 3-dihydro-2-thioxo-4(1H)quinazolinone (R59949) among diacylglycerol kinase subtypes. Biochem Pharmacol 59, 763-772.

Kanoh, H., Yamada, K. \& Sakane, F. (2002). Diacylglycerol kinases: emerging downstream regulators in cell signaling systems. J Biochem 131, 629-633.

Kawada-Matsuo, M., Shibata, Y. \& Yamashita, Y. (2009). Role of two component signaling response regulators in acid tolerance of Streptococcus mutans. Oral Microbiol Immunol 24, 173-176.

Korithoski, B., Lévesque, C. M. \& Cvitkovitch, D. G. (2007). Involvement of the detoxifying enzyme lactoylglutathione lyase in Streptococcus mutans aciduricity. J Bacteriol 189, 7586-7592.

Kremer, B. H., Van der Kraan, M., Crowley, P. J., Hamilton, I. R., Brady, L. J. \& Bleiweis, A. S. (2001). Characterization of the sat operon in Streptococcus mutans: evidence for a role of Ffh in acid tolerance. J Bacteriol 183, 2543-2552.

Lemos, J. A. \& Burne, R. A. (2002). Regulation and physiological significance of ClpC and ClpP in Streptococcus mutans. J Bacteriol 184, 6357-6366.

Lis, M. \& Kuramitsu, H. K. (2003). The stress-responsive $d g k$ gene from Streptococcus mutans encodes a putative undecaprenol kinase activity. Infect Immun 71, 1938-1943.

Loesche, W. J. (1986). Role of Streptococcus mutans in human dental decay. Microbiol Rev 50, 353-380.

Mérida, I., Avila-Flores, A. \& Merino, E. (2008). Diacylglycerol kinases: at the hub of cell signalling. Biochem J 409, 1-18.

Perry, D., Wondrack, L. M. \& Kuramitsu, H. K. (1983). Genetic transformation of putative cariogenic properties in Streptococcus mutans. Infect Immun 41, 722-727.

Preiss, J., Loomis, C. R., Bishop, W. R., Stein, R., Niedel, J. E. \& Bell, R. M. (1986). Quantitative measurement of $s n$-1,2-diacylglycerols 
present in platelets, hepatocytes, and ras- and sis-transformed normal rat kidney cells. J Biol Chem 261, 8597-8600.

Quivey, R. G., Kuhnert, W. L. \& Hahn, K. (2001). Genetics of acid adaptation in oral streptococci. Crit Rev Oral Biol Med 12, 301-314.

Raetz, C. R. \& Newman, K. F. (1979). Diglyceride kinase mutants of Escherichia coli: inner membrane association of 1,2-diglyceride and its relation to synthesis of membrane-derived oligosaccharides. J Bacteriol 137, 860-868.

Rotering, H. \& Raetz, C. R. (1983). Appearance of monoglyceride and triglyceride in the cell envelope of Escherichia coli mutants defective in diglyceride kinase. J Biol Chem 258, 8068-8073.

Sambrook, J. \& Russell, D. W. (2001). Molecular Cloning: a Laboratory Manual, 3rd edn. Cold Spring Harbor, NY: Cold Spring Harbor Laboratory.

Sawatari, Y. \& Yokota, A. (2007). Diversity and mechanisms of alkali tolerance in lactobacilli. Appl Environ Microbiol 73, 3909-3915.

Shibata, Y., Yamashita, Y., Ozaki, K., Nakano, Y. \& Koga, T. (2002). Expression and characterization of streptococcal $r g p$ genes required for rhamnan synthesis in Escherichia coli. Infect Immun 70, 2891-2898.

Shibata, Y., Van der Ploeg, J. R., Kozuki, T., Shirai, Y., Saito, N., Kawada-Matsuo, M., Takeshita, T. \& Yamashita, Y. (2009). Kinase activity of the $d g k$ gene product is involved in the virulence of Streptococcus mutans. Microbiology 155, 557-565.

Tanzer, J. M., Livingston, J. \& Thompson, A. M. (2001). The microbiology of primary dental caries in humans. J Dent Educ 65, 1028-1037.
Topham, M. K. \& Prescott, S. M. (1999). Mammalian diacylglycerol kinases, a family of lipid kinases with signaling functions. J Biol Chem 274, 11447-11450.

Van Blitterswijk, W. J. \& Houssa, B. (2000). Properties and functions of diacylglycerol kinases. Cell Signal 12, 595-605.

Walsh, J. P., Loomis, C. R. \& Bell, R. M. (1986). Regulation of diacylglycerol kinase biosynthesis in Escherichia coli. A trans-acting $d g k R$ mutation increases transcription of the structural gene. J Biol Chem 261, 11021-11027.

Wen, Z. T. \& Burne, R. A. (2004). LuxS-mediated signaling in Streptococcus mutans is involved in regulation of acid and oxidative stress tolerance and biofilm formation. J Bacteriol 186, 26822691.

Wen, Z. T., Suntharaligham, P., Cvitkovitch, D. G. \& Burne, R. A. (2005). Trigger factor in Streptococcus mutans is involved in stress tolerance, competence development, and biofilm formation. Infect Immun 73, 219-225.

Wen, Z. T., Baker, H. V. \& Burne, R. A. (2006). Influence of BrpA on critical virulence attributes of Streptococcus mutans. J Bacteriol 188, 2983-2992.

Yamashita, Y., Takehara, T. \& Kuramitsu, H. K. (1993). Molecular characterization of a Streptococcus mutans mutant altered in environmental stress responses. J Bacteriol 175, 6220-6228.

Yamashita, Y., Tsukioka, Y., Nakano, Y., Tomihisa, K., Oho, T. \& Koga, T. (1998). Biological functions of UDP-glucose synthesis in Streptococcus mutans. Microbiology 144, 1235-1245. 